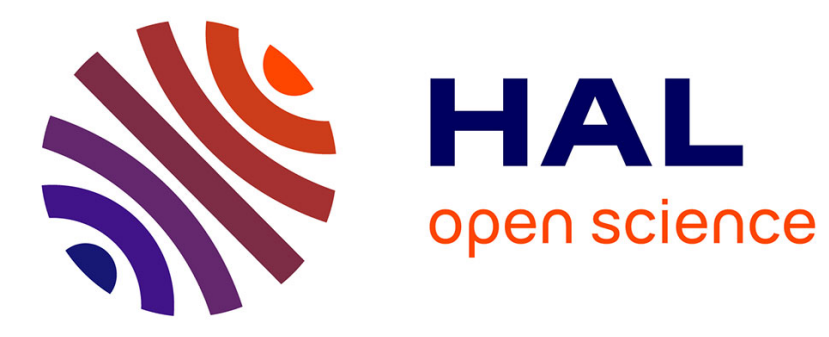

\title{
Towards ultrasound image-based visual servoing
}

\author{
W. Bachta, A. Krupa
}

\section{To cite this version:}

W. Bachta, A. Krupa. Towards ultrasound image-based visual servoing. IEEE Int. Conf. on Robotics and Automation, ICRA'2006, 2006, Orlando, Florida, France. pp.4112-4117. inria-00350281

\section{HAL Id: inria-00350281 https://hal.inria.fr/inria-00350281}

Submitted on 6 Jan 2009

HAL is a multi-disciplinary open access archive for the deposit and dissemination of scientific research documents, whether they are published or not. The documents may come from teaching and research institutions in France or abroad, or from public or private research centers.
L'archive ouverte pluridisciplinaire HAL, est destinée au dépôt et à la diffusion de documents scientifiques de niveau recherche, publiés ou non, émanant des établissements d'enseignement et de recherche français ou étrangers, des laboratoires publics ou privés. 


\title{
Towards Ultrasound Image-Based Visual Servoing
}

\author{
Wael Bachta and Alexandre Krupa \\ IRISA - INRIA Rennes \\ Campus de Beaulieu, 35042 Rennes Cedex, France \\ wael@eavr.u-strasbg.fr, alexandre.krupa@irisa.fr
}

\begin{abstract}
Robotized ultrasound examination offers on one hand more accuracy for the medical diagnostic and on the other hand a suitable assistance to radiologists. In the literature, very few researches deal with ultrasound image-based control of a medical robot holding the ultrasound probe. This paper presents a new visual servoing approach allowing the automatic guidance of the ultrasound probe. This technique is based on the modeling of the coupling between the ultrasound probe and egg shaped objects. The robotic task consists in reaching a desired image from an arbitrary initial position. The aim of this task is to assist the radiologist diagnostic by automatically moving the ultrasound probe in order to find the ultrasound image view of a tumour cross-section registered in a previous date. This work presents simulation results of the proposed ultrasound visionbased control scheme.
\end{abstract}

Index Terms - Visual servoing, ultrasound images, medical robotics.

\section{INTRODUCTION}

Nowadays, the ultrasound imaging is extensively used in medicine. This widespread use is due to the non invasive aspect and the low cost of ultrasound imaging systems. Since few years, several research works have been led to assist the ultrasound examination by the use of medical robots. In [1], a robotic system holding an ultrasound probe is designed. The robotic task consists in moving the ultrasound probe on the patient skin along a preset trajectory. The force applied by the probe to the patient skin is also controlled in order to improve the imaging quality. Other research teams have developed robotic systems for tele-echography purpose (e.g. [2], [3]) which allow the clinicians to perform echographic diagnostics without being present in the examination room. In spite of the numerous developments of robotic systems for ultrasound medical applications, only few robotic systems involve the visual information provided by the ultrasound sensor in their control scheme. Indeed, in the previous mentioned works, the ultrasound probe is only used to acquire the ultrasound images necessary to the medical diagnostic. Nevertheless, in [4], the guidance of a robotic system is shared between an human operator and a vision-based control. This system allows to compensate the physiological motion of an artery during probe displacement along an one-dimensional trajectory. In this work, the ultrasound probe is controlled in three degrees of freedom (DOF) which are constrained to lie in the ultrasound observation plane. In [5], the authors present a robotic system including a motionless ultrasound probe and a two DOF needle manipulator. An automatic needle insertion for a percutaneous cholecystostomy is performed using ultrasound image-based visual servoing. However, in this work, the actuated needle is constrained to lie in the ultrasound observation plane.

Recent studies get rid of the above constraint. In [6], a laparoscopic surgical instrument actuated by a four DOF medical robot is automatically moved to a 3D location thanks to a visual servoing using the ultrasound images provided by a motionless probe. The visual features used in the control scheme are the images coordinates of the points corresponding to the intersection of the instrument with the ultrasound plane. In [7], a visual servoing technique based on ultrasound images is presented to automatically perform the calibration of a robotized 3D ultrasound imaging system. In this work the visual features correspond to the intersection points of a cross-wire object with the ultrasound plane. The weakness of these last works is that objects of interest used to obtain visual information are too restricted (straight lines). In this paper, we propose to consider more complex shaped objects. This implies the use of new modeling techniques, since the ultrasound transducer does not involve any camera perspective projection. Consequently, classical visual servo control features [8] can not be used in our study. Our work is focused on the study of the coupling between the ultrasound plane and egg shaped objects, but it could be extended to any 3D shape that can be modeled by an algebraic curve. The choice of the egg shape is motivated by the likeness of this form to usual tumors.

The paper is organized as follows: the next section introduces the medical motivation of this study and the robotic task to perform. In section III, the ultrasound sensor model is quickly recalled and the geometrical model of the intersection between the ultrasound plane and an egg shaped object is presented. A set of useful visual features is chosen from this modelling and their interaction with the probe motion is derived (section IV). This interaction allows then to develop a new vision-based control. After this modeling issue, a method used to extract the visual features from the ultrasound images is proposed in section V. Results obtained from simulations are presented and discussed in section VI.

\section{ROBOTIC TASK FOR MEDICAL PURPOSE}

In order to assist radiologists, we plan, in further works, to automatically position the ultrasound probe held by a medical robot. The medical application will consist in reaching a tumor cross section having the maximum likelihood with 

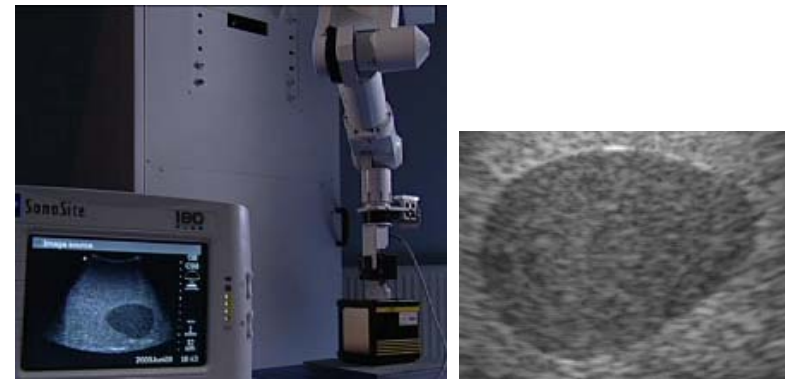

Fig. 1. (a) Test bed including the ultrasound imaging system, an six DOF medical robot holding the ultrasound probe (with a force sensor) and an 3D ultrasound phantom - (b) Ultrasound image of the egg shaped object

a registered one. This will allow the radiologist to analyze quantitatively the tumor evolution using similar 2D ultrasound views captured on different dates.

In order to carry on researches related to ultrasound imagebased visual servoing, we dispose of the test bed shown in fig. 1.a. This experimental setup is composed of an ultrasound imaging system (see section V), a 3D ultrasound phantom (a box containing egg shaped objects which are similar to tumors) and a six DOF medical robot.

In this paper, the robotic task that we propose to accomplish is described in fig.2. The goal is to automatically move the probe in order to reach a desired ultrasound image.

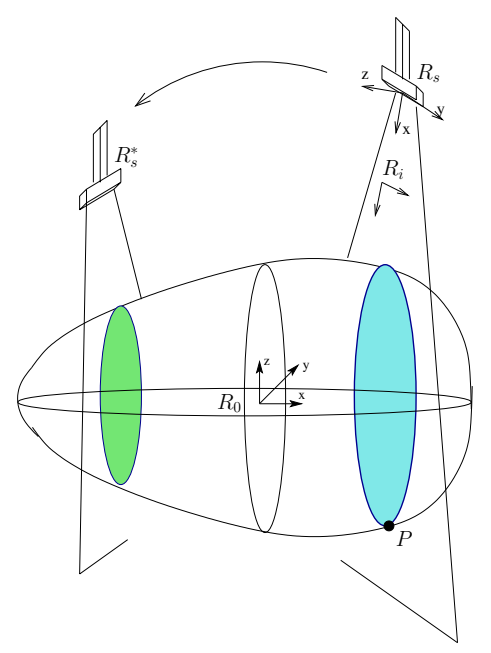

Fig. 2. Robotic task: automatically move the probe to obtain a desired ultrasound image

As mentioned before, we propose to perform it by the means of an ultrasound image-based visual servoing.

\section{GEOMETRICAL MODELING}

The ultrasound image corresponds to the $2 \mathrm{D}$ cross-section of the scene with respect to the observation plane of the ultrasound probe. Modeling this cross-section consists in establishing the equation of the contour that can be extracted from the ultrasound image (see fig 1.b). Let $R_{o}$ and $R_{s}$ be respectively the frames of reference attached to the egg shaped object and the ultrasound probe (see fig. 2). As illustrated in fig. 2, the 2D image frame $R_{i}$ lies in the probe observation plane defined by the $(\mathbf{x}, \mathbf{y})$ plane of $R_{s}$. The coordinates of a point $P$ that belongs to the object and to the observation plane are defined by:

- ${ }^{o} \mathbf{P}=\left[{ }^{o} x,{ }^{o} y,{ }^{o} z\right]^{T}$ in the object frame $R_{o}$

- ${ }^{s} \mathbf{P}=\left[{ }^{s} x,{ }^{s} y, 0\right]^{T}$ in the probe frame $R_{s}$

- ${ }^{i} \mathbf{P}=\left[{ }^{i} x,{ }^{i} y\right]^{T}$ in the 2D image frame $R_{i}$.

The pixel coordinates ${ }^{i} \mathbf{P}$ of the point $P$ expressed in the image frame are obtained from ${ }^{s} \mathbf{P}$ using the following expression :

$$
\left(\begin{array}{c}
{ }^{i} x \\
{ }^{i} y
\end{array}\right)=\left(\begin{array}{cc}
1 / S_{x} & 0 \\
0 & 1 / S_{y}
\end{array}\right)\left(\begin{array}{c}
{ }^{s} x \\
{ }^{s} y
\end{array}\right)+\left(\begin{array}{c}
{ }^{i} x_{c} \\
{ }^{i} y_{c}
\end{array}\right)
$$

where :

- $S_{x}$ and $S_{y}$ are respectively the height and the width of an image pixel (scaling factors of the image)

- ${ }^{i} x_{c}$ and ${ }^{i} y_{c}$ are the pixel coordinates of the image center.

For convenience, we will assume hereafter that $S_{x}=S_{y}=1$ and ${ }^{i} x_{c}={ }^{i} y_{c}=0$. Subsequently, the image coordinates of a point $P$ are equal to those expressed in the probe frame. Therefore, we will not distinguish $\left({ }^{i} x,{ }^{i} y\right)$ and $\left({ }^{s} x,{ }^{s} y\right)$ in the sequel of this paper. Of course, the obtained results can be easily extended to the general case. We will also denote by:

$$
{ }^{s} \mathbf{M}_{o}=\left(\begin{array}{cccc} 
& { }^{s} \mathbf{R}_{o} & & { }^{s} \mathbf{t}_{o} \\
0 & 0 & 0 & 1
\end{array}\right)
$$

the homogeneous transformation matrix that represents the pose between the probe and the object frames. ${ }^{s} \mathbf{R}_{o}$ and ${ }^{s} \mathbf{t}_{o}$ define respectively the rotational matrix and the translational vector from the probe frame to the object frame. Given this matrix, the coordinates of point $P$ expressed in $R_{o}\left({ }^{o} \mathbf{P}\right)$ can be obtained from ${ }^{s} \mathbf{P}$ using the following equation:

$$
{ }^{o} \mathbf{P}={ }^{s} \mathbf{R}_{o}^{T s} \mathbf{P}-{ }^{s} \mathbf{R}_{o}^{T s} \mathbf{t}_{o}
$$

and the previous relation can be written as :

$$
\left\{\begin{aligned}
{ }^{o} x & =f_{1}\left({ }^{s} \mathbf{M}_{o},{ }^{s} x,{ }^{s} y\right) \\
{ }^{o} y & =f_{2}\left({ }^{s} \mathbf{M}_{o},{ }^{s} x,{ }^{s} y\right) \\
{ }^{o} z & =f_{3}\left({ }^{s} \mathbf{M}_{o},{ }^{s} x,{ }^{s} y\right)
\end{aligned}\right.
$$

where $f_{1}, f_{2}$ and $f_{3}$ are linear functions of ${ }^{s} x$ and ${ }^{s} y$.

If we choose an adequate object frame, the egg shaped object can be modeled by the following algebraic equation (obtained by modifying the ellipsoid equation):

$$
\frac{{ }^{o} x^{2}}{a^{2}}+\frac{{ }^{o} y^{2}}{b^{2}}\left(1+\frac{d^{2}+2 d x}{a^{2}}\right)+\frac{{ }^{o} z^{2}}{c^{2}}\left(1+\frac{d^{2}+2 d x}{a^{2}}\right)=1
$$

with $(a, b, c)$ the egg parameters that we denote by $\mathcal{S}$.

By substituting (4) in (5), we obtain :

$$
\sum_{i, j \geq 0, i+j \leq 3} a_{i j}{ }^{s} x^{i}{ }^{s} y^{j}=0
$$


with :

$$
\left[a_{i j}\right]_{i, j \geq 0, i+j \leq 3}=\left[f_{i j}\left(\mathcal{S},{ }^{s} \mathbf{M}_{o}\right)\right]_{i, j \geq 0, i+j \leq 3}
$$

So, the contour representing the intersection between the ultrasound observation plane and the egg shaped object can be defined as a third order polynomial.

\section{VISUAL SERVOING}

The robotic task can be formulated as a regulation problem. The goal consists in moving by visual servoing [9] the probe such as pertinent visual features reaches desired ones. In this section, a useful set of visual features is first chosen. Then the interaction matrix, that links the image features variation to the relative kinematic screw between the probe and the scene, is determined. In our approach all the parameters required to the calculus of this matrix will be estimated. Finally an adequate control law is given.

\section{A. Interaction Matrix}

Since we obtain the image of different 3D points whenever the ultrasound sensor moves, it doesn't seem obvious to try to establish an image point interaction matrix. Thus, nice visual features like image moments [10] can not be simply employed in the current study. In fact, the interaction matrices of these primitives are directly derived from a point interaction matrix by applying the Green theorem [10].

As all the images of the egg represent a curve that can be modeled by a third order polynomial (6), we propose to take the set of polynomial coefficients as visual features.

However this representation of the algebraic curve is ambiguous. Indeed, the multiplication of all the coefficients by a nonnull real doesn't modify the contour shape. To cope with this problem, the equation (6) is rewritten as :

$$
{ }^{s} x^{3}+\sum_{i, j \geq 0, i+j \leq 3, i<3} p_{i j}{ }^{s} x^{i}{ }^{s} y^{j}=0
$$

with :

$$
\mathbf{s}=\left[p_{i j}\right]_{i, j \geq 0, i+j \leq 3, i<3}=\left[\frac{a_{i j}}{a_{30}}\right]_{i, j \geq 0, i+j \leq 3, i<3}
$$

Here $\mathbf{s}$ is the considered visual features vector we retain. Now let us calculate the interaction matrix $\mathbf{L}_{s}$ such as :

$$
\dot{\mathbf{s}}=\mathbf{L}_{s} \mathbf{v}
$$

with $\mathbf{v}=[\boldsymbol{v}, \boldsymbol{\omega}]^{T}$ the velocity screw of the probe frame expressed in $R_{s} . \boldsymbol{v}=\left[v_{x}, v_{y}, v_{z}\right]^{T}$ and $\boldsymbol{\omega}=\left[\omega_{x}, \omega_{y}, \omega_{z}\right]^{T}$ are respectively the translational and the angular velocity vectors. In order to establish $\mathbf{L}_{s}$, the time derivative of the image features vector $\mathbf{s}$ is calculated:

$$
\dot{\mathbf{s}}=\left[\dot{p}_{i j}\right]_{i, j \geq 0, i+j \leq 3, i<3}=\left[h_{i j}\left(\mathcal{S},{ }^{s} \mathbf{M}_{o}, \dot{\mathbf{M}}_{o}\right)\right]
$$

where $h_{i j}$ are function of $\mathcal{S},{ }^{s} \mathbf{M}_{o}$ and ${ }^{s} \dot{\mathbf{M}}_{o} \cdot{ }^{s} \dot{\mathbf{M}}_{o}$ is obtained using the following well known kinematic fundamental laws :

$$
\left\{\begin{array}{ccc}
{ }^{s} \dot{\mathbf{R}}_{o} & =-[\boldsymbol{\omega}]_{\times}{ }^{s} \mathbf{R}_{o} \\
{ }^{s} \dot{\mathbf{t}}_{o} & =-\boldsymbol{v}+\left[{ }^{s} \mathbf{t}_{o}\right]_{\times} \boldsymbol{\omega}
\end{array}\right.
$$

where $[\boldsymbol{\omega}]_{\times}$and $\left[{ }^{s} \mathbf{t}_{o}\right]_{\times}$are the skew symmetric matrices associated respectively with $\boldsymbol{\omega}$ and ${ }^{s} \mathbf{t}_{o}$.

By employing (12) in (11), we obtain the analytical form of the interaction matrix such as:

$$
\dot{\mathbf{s}}=\left[\dot{p}_{i j}\right]_{i, j \geq 0, i+j \leq 3, i<3}=\mathbf{L}_{s}\left(\mathcal{S},{ }^{s} \mathbf{M}_{o}\right) \mathbf{v}
$$

The condition to compute $\mathbf{L}_{s}$ is that $a_{30} \neq 0$. For a lake of place, the matrix components are not given here.

\section{B. Pose estimation}

The interaction matrix $\mathbf{L}_{s}$ depends on the egg model $\mathcal{S}$ and the homogeneous matrix ${ }^{s} \mathbf{M}_{o}$. The model parameters of the egg shaped object $\mathcal{S}$ are assumed to be provided. However, ${ }^{s} \mathbf{M}_{O}$ is not known and has to be estimated. It can be written as :

$$
{ }^{s} \mathbf{M}_{o}={ }^{s} \mathbf{M}_{b}{ }^{b} \mathbf{M}_{o}
$$

with :

- ${ }^{s} \mathbf{M}_{b}$ the homogeneous matrix representing the pose between the probe and the robot base frames.

- ${ }^{b} \mathbf{M}_{o}$ the constant homogeneous matrix representing the pose between the robot base and the object frames.

This decomposition allows us to estimate constant parameters instead of varying ones. Indeed, ${ }^{s} \mathbf{M}_{b}$ corresponds to the robot geometric model provided by the robot controller. Consequently, only the constant matrix ${ }^{b} \mathbf{M}_{o}$ has to be estimated. As we can see in equations (7) and (9), the visual features vector elements also depend on ${ }^{s} \mathbf{M}_{o}$. These elements can be expressed in terms of ${ }^{b} \mathbf{M}_{o}$ coefficients using (14).

If the rotation of $R_{o}$ with respect to the robot base frame $R_{b}$ is described by the minimal representation $\theta u$, we can represent the homogeneous matrix ${ }^{b} \mathbf{M}_{O}$ by a 6 dimension vector $\mathbf{x}$ including a translational vector and a $\theta u$ angle vector. Therefore, it can be written :

$$
\left.p_{i j}\right]_{i, j \geq 0, i+j \leq 3, i<3}=\left[l_{i j}\left(\mathcal{S},{ }^{s} \mathbf{M}_{b}, \mathbf{x}\right)\right]_{i, j \geq 0, i+j<3, i<3}
$$

where $l_{i j}$ are function of $\mathcal{S},{ }^{s} \mathbf{M}_{b}$ and $\mathbf{x}$. As mentioned previously, $\mathcal{S}$ and ${ }^{s} \mathbf{M}_{b}$ are provided, so we can rewrite (15) by:

$$
\left[p_{i j}\right]_{i, j \geq 0, i+j \leq 3, i<3}=\left[m_{i j}(\mathbf{x})\right]_{i, j \geq 0, i+j \leq 3, i<3}
$$

The $p_{i j}$ can be directly computed from the ultrasound image. So, computing the pose consists in solving the previous nonlinear 9 equations system. As the pose to estimate is constant, many images can be used to determine $\mathbf{x}$. If we 
denote $g_{i j}=p_{i j}-m_{i j}$, we have to solve the following nonlinear equations system in order to estimate the pose.

$$
\mathbf{g}(\mathbf{x})=\left[g_{i}(\mathbf{x})\right]_{1 \leq i \leq 9 N}=\overrightarrow{0}
$$

where $N$ is the number of images used in the estimation. This system can be solved iteratively by finding the solution of the following least squares problem :

$$
\mathbf{x}^{*}=\operatorname{argmin}_{x}\left\{G(\mathbf{x})=\frac{1}{2} \mathbf{g}(\mathbf{x}) \mathbf{g}^{T}(\mathbf{x})\right\}
$$

We propose to solve this problem by means of the LevenbergMarquardt algorithm. This algorithm implies a damping parameter that allows us to get the robustness of the steepest descent method when $\mathbf{x}$ is far from $\mathbf{x}^{*}$ and the faster convergence of the Gauss-Newton technique if $\mathbf{x}$ is close to the solution. We use the strategy proposed in [11] to update the damping parameter.

In practice, the following estimation process is applied: at each iteration $k$ of the visual servoing loop, the solution $\mathbf{x}^{*}$ obtained in the precedent iteration is used as a starting point. The images acquired in the $k-1$ and the $k$ iterations are used to process the estimation. In order to have a total control on the processing time, the number of iterations is restricted to 10 .

Using the proposed algorithm, the interaction matrix can be fully estimated on-line.

\section{Control laws}

The vision-based task function approach [12] is adopted to perform the robotic task. The control problem can be expressed as a regulation to zero of the following task function [9]:

$$
\mathbf{e}=\mathbf{C}\left(\mathbf{s}-\mathbf{s}^{*}\right)
$$

where :

- $\mathbf{s}$ is the current visual features vector (9 dimension) measured from the ultrasound image,

- $\mathbf{s}^{*}$ is the reference image features vector (9 dimension) to be reached,

- $\mathbf{C}$ is a $6 \times 9$ combination matrix. A judicious choice of $\mathbf{C}$ is $\mathbf{C}=\widehat{\mathbf{L}}_{\left.s\right|_{s=s^{*}}}^{+}$with $\widehat{\mathbf{L}}_{\left.s\right|_{s=s^{*}} ^{+}}$an approximation of the pseudo inverse interaction matrix at the desired position (see [9]).

Generally, the control law is defined such as the task e decreases exponentially and is given by [9]:

$$
\mathbf{v}=-\lambda\left(\widehat{\mathbf{L}}_{\left.s\right|_{s=s^{*}} ^{+}} \widehat{\mathbf{L}_{s}}\right)^{-1} \mathbf{e}
$$

where :

- $\lambda$ is a proportional coefficient involved in the exponential convergence of $\mathbf{e}$,

- $\widehat{\mathbf{L}_{s}}$ is an approximation of the current interaction matrix. The on-line estimation method is presented in IV.B,

- $\widehat{\mathbf{L}}_{\left.s\right|_{s=s^{*}} ^{+}}^{+}$is an approximation of the pseudo inverse interaction matrix at the desired position.
A second-order minimization technique can also be used [13] in order to improve the probe trajectory in the 3D space (a straight line for the translation and a rotation around the axis of rotation).

The corresponding control law becomes then:

$$
\begin{gathered}
\mathbf{v}=-\frac{\lambda}{2}\left(\widehat{\mathbf{L}}_{\left.s\right|_{s=s^{*}} ^{+}}^{+}\left(\widehat{\mathbf{L}_{s}}+\widehat{\mathbf{L}_{s \mid s=s^{*}}}\right)\right)^{-1} \mathbf{e} \\
\text { V. IMAGE PROCESSING }
\end{gathered}
$$

\section{A. Imaging system}

The ultrasound imaging system is composed of a SonoSite 180PLUS (2D imaging system) connected to a broadband 5$10 \mathrm{MHz}$ curvilinear probe. A Pentium IV PC running under a Linux operating system is used to grab via a capture board gray level $576 \times 768$ images at $25 \mathrm{fps}$ and to perform the image processing. The figure 8 shows the ultrasound image of a $2 \mathrm{D}$ cross section of the egg shaped object included in the ultrasound phantom. The first step consists in extracting a set of contour points from the image. Then the polynomial coefficients of the curve are estimated from these points by using a least-squares algorithm.

\section{B. Visual features extraction}

For real-time purpose, only a sub-block (which contains the contour) of the acquired image is processed. In each frame a first Gaussian filter is applied to the whole defined sub-block image in order to reduce the speckle noise. Then 20 angular equidistant radius are projected from a point positioned inside the contour. This point is the center of mass of the contour points obtained in the previous image. As the image is still noisy, a second Gaussian mask is applied to all the pixels along each radius. Then a mono-directional gradient is computed from the grey level of the considered points. Finally, for each radius, the pixel point that presents the highest gradient value is retained as a contour point of the object image. In order to estimate the polynomial coefficients from the extracted contour points, the "Gradient-one Fitting" [14] algorithm is used.

\section{RESUlTS}

\section{A. Visual servoing}

The results of the proposed control laws (20) and (21) are described and discussed in this section. Only simulation results are given but we plan to perform our task with a 6 DOF medical robot specially designed for 3D ultrasound imaging. A software simulator was programmed in Matlab environment from the geometrical modeling described in section III. The parameters for the egg shaped object are $\mathcal{S}(a=2, b=3, c=$ $2.5, d=0.7)(\mathrm{cm})$. Hereafter, the roll-pitch-yaw representation is used to describe rotations.

The first task to perform is shown in fig.3. In fact the goal is to automatically move the ultrasound probe such as the original contour (colored in red) reaches the desired one (colored in blue). The displacement required from the ultrasound probe is a translation of $[2.0,0.3,0.2]^{T}(\mathrm{~cm})$ and a rotation of $[10,20,-5]^{T}($ deg $)$.

In this simulation a comparison between the two control 
laws ((20) and (21)) presented in paragraph IV.C is achieved. This comparison highlights the advantages of the second-order minimization technique given in (21). In one hand we can easily see on fig.4.b that the 3D translation movement is almost a straight line and on the other hand a poor 3D behaviour is observed when using the classical control law (fig.4.a). Moreover the use of the efficient control law provides more decoupled 3D movements which can be seen on the velocity screw response (fig.4.b). As optimal 3D displacements are necessary in our future medical application, the second order minimization based control law is then retained.

The figure 5 shows a second robotic task to perform. The required displacement is defined by a translation of $[1.6,-0.5,-0.4]^{T}(\mathrm{~cm})$ and a rotation of $[10,20,15]^{T}(\mathrm{deg})$. For this task the homogeneous matrix ${ }^{b} M_{o}$ is defined by ${ }^{o} R_{b}(-10,-10,-10)(\mathrm{deg})$ and $\left.{ }^{o} t_{b}=[-1,-1,-1](\mathrm{cm})\right)$. During the simulation we put significant error on the initial value of ${ }^{b} M_{o}$ (we set ${ }^{o} R_{b}(5,10,20)(\mathrm{deg})$ and ${ }^{o} t_{b}=$ $[-0.5,-0.5,-0.5](\mathrm{cm}))$ and the visual servoing is performed using the on-line estimation described in paragraph IV.B. The simulation results related to this task are given in fig.6. The convergence of the task function and the visual features error are shown in fig.6.a. We can remark that the propsed task is performed even with the set error. Therefore our visual servoing is robust to the initial errors on ${ }^{b} M_{o}$. As seen in fig.6.b, the on-line estimation of the pose $\mathrm{x}$ converges to real value.

The same task was achieved a second time. But in the last simulation, we not only set an error on ${ }^{b} M_{o}$ (with ${ }^{o} R_{b}(5,10,20)(\mathrm{deg})$ and $\left.{ }^{o} t_{b}=[-0.5,-0.5,-0.5](\mathrm{cm})\right)$ but also simulate some noise on the current visual features (the equivalent of $+/-1$ pixel assuming that a pixel is a square of $10^{-2} \mathrm{~cm} \times 10^{-2} \mathrm{~cm}$ ). This additional noise allows us to test the "Gradient-one-fitting" algorithm mentionned in paragraph V.B while processing the visual servoing loop. The results presented in fig. 7 show the robustness of our visual servoing to these measurement disturbances.

The obtained simulation results are promising, so the next step in this work is to validate them experimentally. One important issue is to study the robustness of the proposed visual servoing scheme to the model errors. In fact a tumour can only be approximately fitted by a third order polynomial. One can use higher order polynomials to have more accurate modeling, but this will create more local minima. So other geometrical representations like splines could be considered.

\section{B. Image processing}

The ultrasound images segmentation is a well visited field and many robust algorithms are available in the litterature. Nevertheless, when real time processing constraints are in concern the existent methods are generally limited.

That's why we have propopsed a simple way to extract the necessary visual features. Figure 8 represents the curves obtained using the contour detection algorithm and the polynomial fitting technique proposed in section V. The processing time of each image is about $30 \mathrm{~ms}$. So it can be performed in real- time for a standard image rate of $25 \mathrm{fps}$. Moreover we dispose of $10 \mathrm{~ms}$ to compute the control law.

The obtained result is satisfying. However, the segmented images have a relatively good quality compared to in-vivo ones. So, the robustness of the proposed segmentation technique has to be extensively tested.

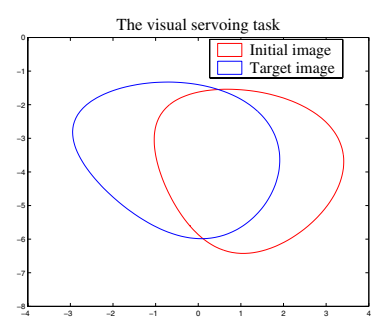

Fig. 3. The first task to perform
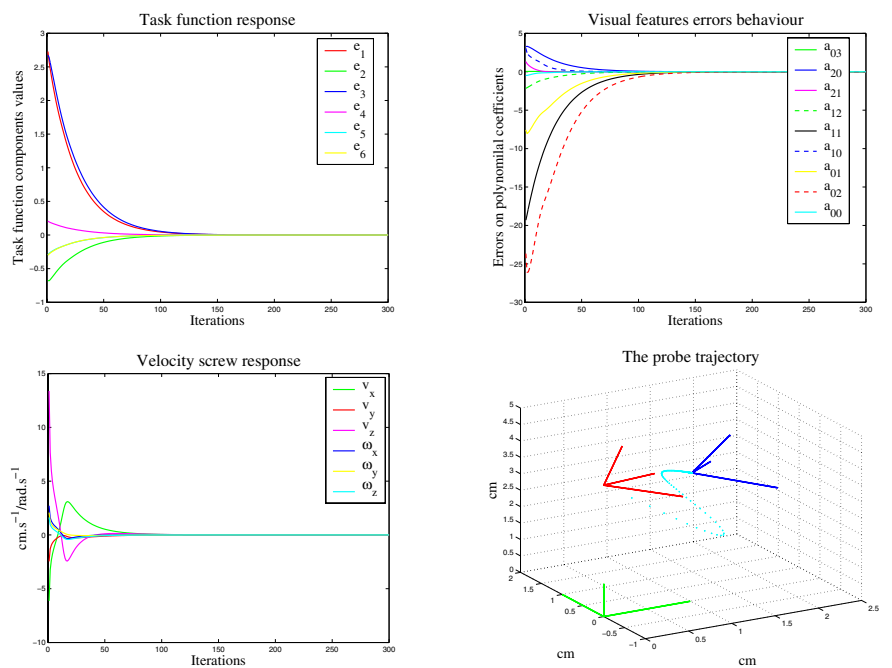

(a) Results using the classical control law given in (20)
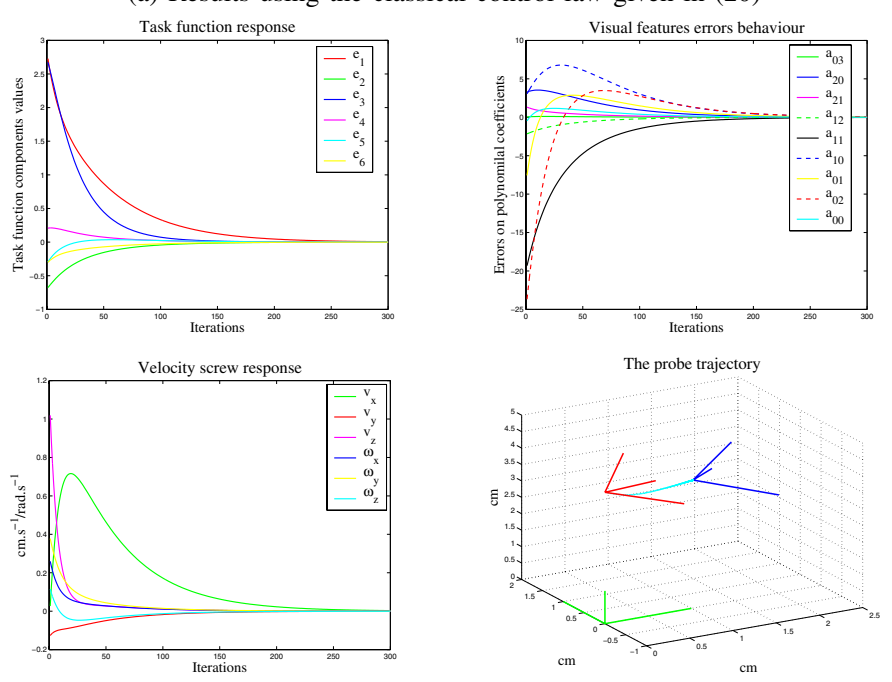

(b) Results using the second order minimization technique given in (21)

Fig. 4. Results of the comparison between the classical control law and second order minimization technique 


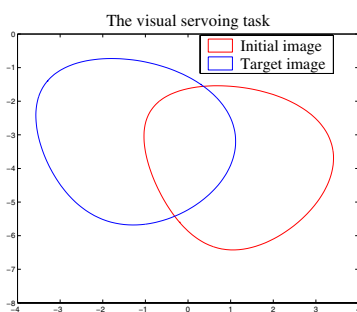

Fig. 5. The second task to perform
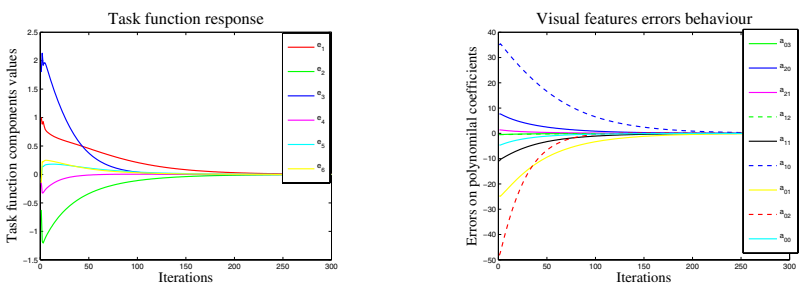

(a) Results using the estimation technique
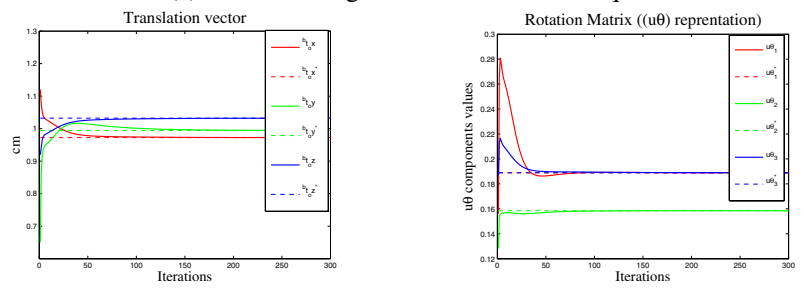

(b) Pose estimation

Fig. 6. The visual servoing robustness to initial modeling errors when using the second order minimization technique given in (21)
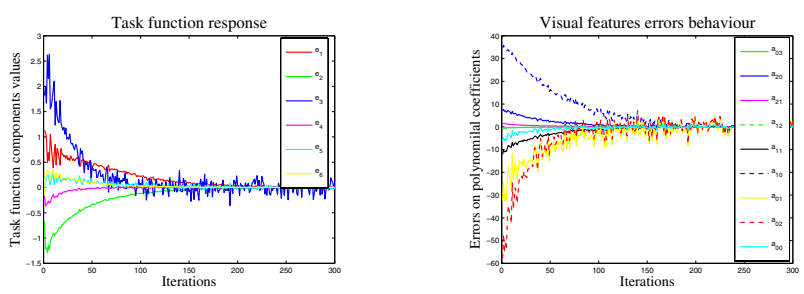

Fig. 7. The visual servoing robustness to the image noise
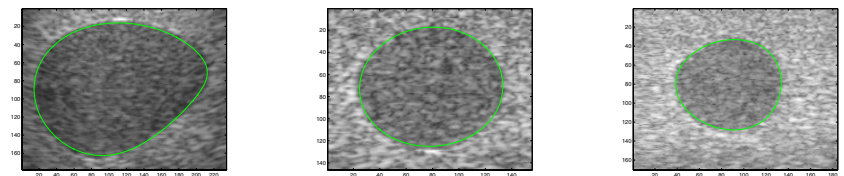

Fig. 8. Image processing results : the estimated contour on the ultrasound image

\section{CONCLUSION}

In this paper, an original ultrasound image-based visual servoing was proposed. The considered task consists in automatically moving the ultrasound probe in order to reach a desired image from an arbitrary initial position. In our approach a set of complex visual features was defined and the corresponding interaction matrix was derived from the geometrical model of the ultrasound system. All the unknown parameters necessary to compute this matrix were estimated. Two adaptive control laws, with an on-line pose estimation technique, were tested by simulation. A method to extract the visual features was also given. As the simulation results are promising, we plan to validate experimentally this ultrasound image-based visual servoing by using a medical robot. A hybrid force/vision control scheme will also be developed in order to control the force necessary to keep the probe on the patient skin in order to perform robotized medical applications. One can imagine other medical applications such as automatically finding the tumor largest section in order to evaluate the pathology characteristics.

\section{REFERENCES}

[1] F. Pierrot, E. Dombre, E. Degoulange, L. Urbain, P. Caron, S. Boudet, J. Gariepy, and J. Megnien, "Hippocrate : A safe robot arm for medical applications with force feedback," Medical Image Analysis (MedIA), Special Issue on Medical Robotics and Computer Assisted Surgery, vol. 3, no. 3, pp. 285-300, 1999.

[2] K. Masuda, E. Kimura, N. Tateishi, and K. Ishihara, "Three dimensional motion mechanism of ultrasound probe and its application for teleechography system," in Proc. IEEE/RSJ Int. Conf. Intelligent Robots and Systems, 2001, pp. 1112-1116.

[3] A. Vichlis, J. Troccaz, P. Cinquin, K. Masuda, and F. Pellisier, "A new robot architecture for tele-echography," IEEE Trans. Robot. Automat., vol. 19, no. 5, pp. 922-926, 2003.

[4] P. Abolmaesumi, S. Salcudea, W. Zhu, M. Sirouspour, and S. DiMaio, "Image-guided control of a robot for medical ultrasound," IEEE Trans. Robot. Automat., vol. 18, pp. 11-23, 2002.

[5] J.Hong, T. Dohi, M. Hashizume, K. Konishi, and N. Hata, "An ultrasound-driven needle insertion robot for percutaneous cholecystostomy," Physics in Medicine and Biology, vol. 49, no. 3, pp. 441-455, 2004.

[6] M. Vitrani, G. Morel, and T. Ortmaier, "Automatic guidance of a surgical instrument with ultrasound based visual servoing," in Proc. IEEE Int. Conf. on Robotics and Automation, Barcelona, Spain, April 2005.

[7] A. Krupa and F. Chaumette, "Control of an ultrasound probe by adaptive visual servoing," in IEEE/RSJ Int. Conf. Intelligent Robots and Systems, Edmonton, Alberta Canada, August 2005.

[8] S. Hutchinston, G. Hager, and P. Corke, "A tutorial on visual servo control," IEEE Trans. on Robotics and Automation, vol. 12, no. 5, pp. 651-670, October 1996.

[9] B. Espiau, F. Chaumette, and P. Rives, "A new approach to visual servoing in robotics," IEEE Trans. Robot. Automat., vol. 8, no. 3, pp. 313-326, 1992.

[10] F. Chaumette, "Image moments: a general and useful set of features for visual servoing," IEEE Trans. on Robotics, vol. 20, no. 4, pp. 713-723, August 2004.

[11] H. B. Nielsen, "Damping parameter in marquardt's method," Department of mathematical modelling, Technical University of Denmark, Tech. Rep. IMM-REP-1999-05, 1999.

[12] C. Samson, M. L. Borgne, and B. Espiau, "Robot control : The task function approach," Clarendon Press, Oxford, United Kingdom, 1991.

[13] E. Malis, "Improving vision-based control using efficient second-order minimization techniques," in Proc. IEEE Int. Conf. on Robotics and Automation, New Orleans, USA, April 2004.

[14] T. Tasdizen, J.-P. Tarel, and D. Cooper, "Improving the stability of algebraic curves for applications," IEEE Transactions on Image Processing, vol. 9, no. 3, pp. 405-416, March 2000. 